\title{
Shot noise as a probe of spin-polarized transport through single atoms
}

\author{
Burtzlaff, Andreas; Weismann, Alexander; Brandbyge, Mads; Berndt, Richard
}

Published in:

Physical Review Letters

Link to article, DOI:

10.1103/PhysRevLett.114.016602

Publication date:

2015

Document Version

Publisher's PDF, also known as Version of record

Link back to DTU Orbit

Citation $(A P A)$ :

Burtzlaff, A., Weismann, A., Brandbyge, M., \& Berndt, R. (2015). Shot noise as a probe of spin-polarized transport through single atoms. Physical Review Letters, 114(1), 016602.

https://doi.org/10.1103/PhysRevLett.114.016602

\section{General rights}

Copyright and moral rights for the publications made accessible in the public portal are retained by the authors and/or other copyright owners and it is a condition of accessing publications that users recognise and abide by the legal requirements associated with these rights.

- Users may download and print one copy of any publication from the public portal for the purpose of private study or research.

- You may not further distribute the material or use it for any profit-making activity or commercial gain

- You may freely distribute the URL identifying the publication in the public portal 


\title{
Shot Noise as a Probe of Spin-Polarized Transport through Single Atoms
}

\author{
Andreas Burtzlaff, ${ }^{1}$ Alexander Weismann, ${ }^{1}$ Mads Brandbyge, ${ }^{2}$ and Richard Berndt ${ }^{1}$ \\ ${ }^{1}$ Institut für Experimentelle und Angewandte Physik, Christian-Albrechts-Universität zu Kiel, D-24098 Kiel, Germany \\ ${ }^{2}$ DTU Nanotech Department of Micro and Nanotechnology, NanoDTU, Technical University of Denmark, \\ DK-2800 Kongens Lyngby, Denmark
}

(Received 20 August 2014; revised manuscript received 10 November 2014; published 6 January 2015)

\begin{abstract}
Single atoms on $\mathrm{Au}(111)$ surfaces have been contacted with the Au tip of a low temperature scanning tunneling microscope. The shot noise of the current through these contacts has been measured up to frequencies of $120 \mathrm{kHz}$ and Fano factors have been determined to characterize the transport channels. The noise at Fe and Co atoms, the latter displaying a Kondo effect, indicates spin-polarized transport through a single channel. Transport calculations reproduce this observation.
\end{abstract}

DOI: 10.1103/PhysRevLett.114.016602

The influence of the electron spin on the electrical current through a nanostructure may be probed by measuring its magnetoresistance. A less common approach is to analyze the shot noise of the current, which results from the discreteness of the electron charge. Schottky derived the power spectral density [1] of this white noise, $S_{0}=2 e I$, for vacuum diodes [3]. In a quantum system the Pauli principle results in antibunching of electrons with identical spins [4]. This reduces the noise, which in turn may be used to determine the degree of spin polarization of the electrons.

We implemented noise spectroscopy in a low temperature scanning tunneling microscope (STM) to probe spin effects in the transport through single atoms and molecules without requiring a magnetic tip, as used in a spin-polarized STM [5]. Here we report data from single atoms on a $\mathrm{Au}(111)$ single crystal surface. We verified that the noise from $\mathrm{Au}$ atoms is consistent with previous reports [6,7]. On $\mathrm{Fe}$ atoms, however, the shot noise is significantly reduced below the minimum value for spin-degenerate transport. On Co atoms a similar reduction is observed, although the Kondo effect, screening of the localized spin by band electrons, occurs. We interpret these findings as being due to a spin polarization of the transmission through a single magnetic atom. Spin-polarized transport calculations neglecting spin-orbit coupling and electron-electron interactions beyond the mean field approximation reproduce the experimental results.

Noise spectroscopy of mesoscopic structures has been reviewed in Ref. [8]. As to atomic scale structures, some results for the tunneling range (conductance $G \lesssim 0.043 G_{0}$, $\left.G_{0}=2 e^{2} / h\right)$ have been reported from STM studies [9-11]. In break junctions shot noise has been investigated for atomic $\mathrm{Au}$ [6,7,12], $\mathrm{Al}$ [13], and $\mathrm{Pt}$ [14] contacts with $G \gtrsim G_{0}$ as well as for benzene [15]. It has been probed at optical frequencies for $\mathrm{Ag}$ atoms [16] and $\mathrm{C}_{60}$ molecules [17]. Atomic Pt wires, which are expected to exhibit spinpolarized transport, have been investigated, but no experimental evidence of spin polarization was observed [18].
PACS numbers: 72.25.Mk, 72.70.+m, 73.40.Jn, 74.55.+v

We used a low temperature STM operated at $4.5 \mathrm{~K}$ in ultrahigh vacuum (UHV). Au(111) single crystal surfaces were prepared by cycles of Ar sputtering and annealing. Tips were etched from $\mathrm{W}$ wire and prepared in UHV by annealing and Ar ion bombardment. After mounting into the STM, they were indented into the Au crystal to coat their apex with gold. Finally, the sample was contacted until single $\mathrm{Au}$ atoms were deposited from the tip and the contacts were stable with $G \approx G_{0}$. Moreover, the presence of the $\mathrm{Au}(111)$ surface state in $d I / d V$ spectra was verified.

After tip preparation we deposited single Co or Fe atoms on the cold sample using electron beam evaporation. $d I / d V$ spectra of Co atoms showed a Kondo resonance [19] and an apparent height of $\approx 130 \mathrm{pm}$. Fe atoms exhibited a similar height but no Kondo signature. Contact was achieved by positioning the STM tip above an adatom, switching off the feedback loop, and bringing the tip closer until a jump was observed in the currentdistance curve, $I(\Delta z)$, which was recorded simultaneously [Figs. 1(a)-1(c)]. At a sample voltage of $128 \mathrm{mV}$, contact conductances between $0.9-1.0 G_{0}$ for Au [Fig. 1(a)] and $\approx 0.8 G_{0}$ for Co [Fig. 1(b)] were found. The range of conductances measured at the same voltage on the $\mathrm{Fe}$ contacts was considerably broader, ranging from 0.47 to around $0.68 G_{0}$ [Fig. 1(c)].

For noise measurements the junction was disconnected from the STM electronics using relays and biased at a constant current from a battery driven power supply. The voltage noise of the contact was measured by two homebuilt differential amplifiers in parallel and was sampled using a storage oscilloscope. By cross-correlating both signals, the uncorrelated input voltage noise of the amplifiers was strongly suppressed [20]. The spectra were corrected for a low-pass roll-off due to the contact impedance and cabling capacitance $\left(f_{-3 \mathrm{~dB}}=60 \mathrm{kHz}\right.$ at $G=0.5 G_{0}$ ), similar to Ref. [6]. The acquisition time for a noise spectrum at a single bias current was $\approx 3$ minutes. The dc current was measured by switching a transimpedance 

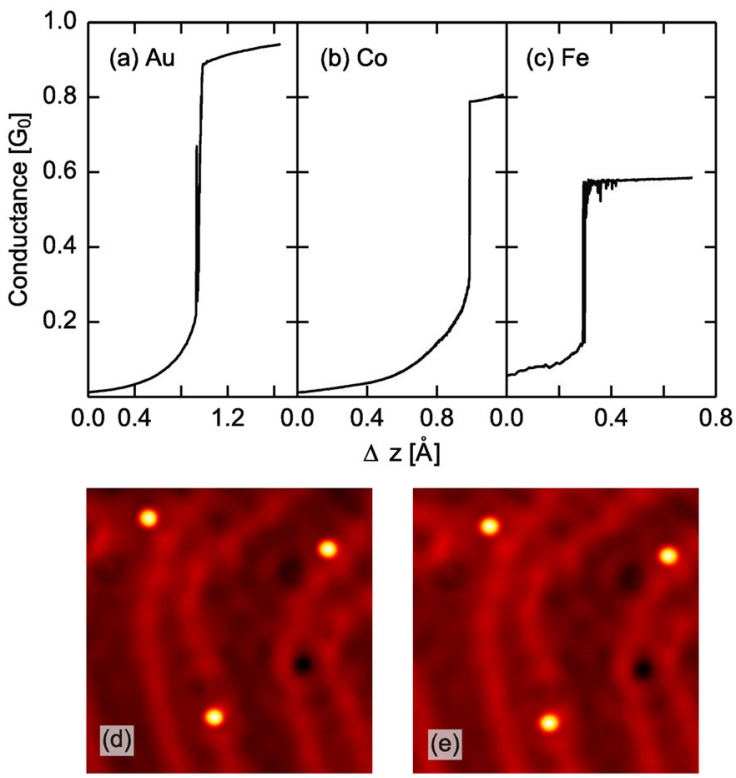

FIG. 1 (color online). Conductance vs displacement of the tip towards the sample for contacts to single (a) $\mathrm{Au}$, (b) $\mathrm{Co}$, and (c) $\mathrm{Fe}$ atoms, measured at a sample voltage of $128 \mathrm{mV}$. Topographs [128 mV, $\left.0.1 \mathrm{nA},(13 \mathrm{~nm})^{2}\right]$ of three Fe atoms (d) before and (e) after performing a noise measurement show no modifications.

amplifier into the circuit before and after each noise measurement.

The lateral position of the tip with respect to the adatom turned out to be important, as small tip displacements resulted in fluctuations of the adatom between hexagonalclose-packed and face-centered-cubic sites, as reported earlier [21,22]. To detect this and other detrimental effects, the current through the contact was ramped up and down with noise data being recorded at an interleaved set of currents, and topographs were recorded before and after noise measurements. Figures 1(d) and 1(e) show an example of three $\mathrm{Fe}$ atoms. Noise data were recorded on the lower one. This atom, its local environment, and the imaging properties of the tip did not change notably. Below we only present data of the contacts that remained stable.

Figure 2 displays examples of the spectral current noise densities of a $0.96 G_{0} \mathrm{Au}$ and a $0.66 G_{0} \mathrm{Fe}$ contact for identical bias currents. At $I=0$, the spectra show white noise, while $1 / f$ noise is present at low frequencies for $I>0$. At higher frequencies, however, the spectra are white and the noise levels increase with the current, as expected for shot noise. The increase with current is significantly larger for the $\mathrm{Fe}$ atom than for the $\mathrm{Au}$ one. To quantify the behavior, we calculated the excess noise $\Delta S$ by subtracting the $I=0$ spectrum, as this contains thermal noise $S_{\Theta}=$ $4 k_{B} T G$ and correlated contributions originating from the amplifier's input current noise. The spectral density was then averaged over a frequency interval (110 to $120 \mathrm{kHz})$ where white noise was present. Figure 3 shows the results as a function of bias current for a number of different atoms and both bias polarities.

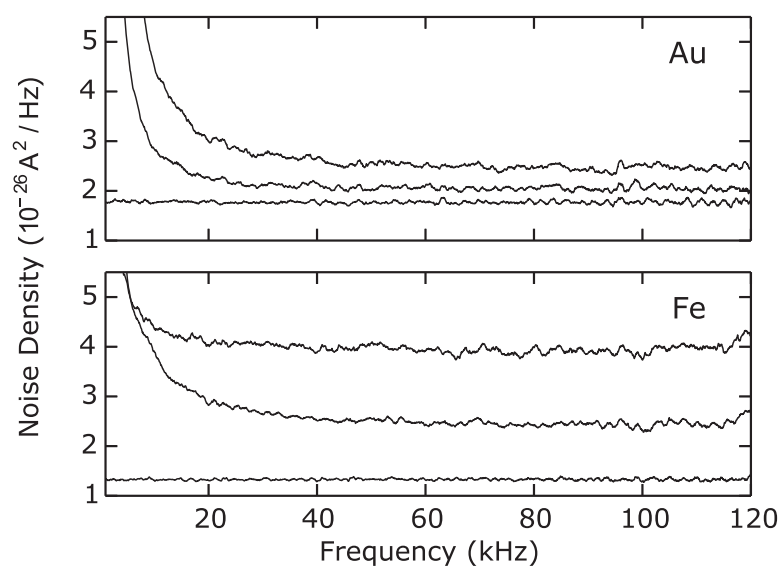

FIG. 2. Current noise density corrected for frequency response at $\mathrm{Au}$ and $\mathrm{Fe}$ adatoms for bias currents 0 (lowest spectra), 0.17, and $0.34 \mu \mathrm{A}$. For the Fe atom the noise spectral density increases significantly faster with the current than for the $\mathrm{Au}$ atom. A moving average filter has been applied for ease of comparison.

To interpret the results, we use the Landauer-Büttiker model. The current is carried by conduction channels with transmission probabilities $\tau_{i}$ leading to a total conductance $G=G_{0} \sum_{i} \tau_{i}$ [23]. When the spin degeneracy of these channels is lifted, it is useful to introduce spin channels $j=(i, \sigma)$ where $\sigma$ is the spin. Compared to the Poissonian noise $S_{0}$, electron correlations reduce the shot noise spectral density $S$ by the Fano factor $F$ :

$$
F=\frac{\sum_{j} \tau_{j}\left(1-\tau_{j}\right)}{\sum_{j} \tau_{j}}
$$

Thus the Fano factor provides insight into the transparencies $\tau_{i}$ of the transport channels and their spin polarization.

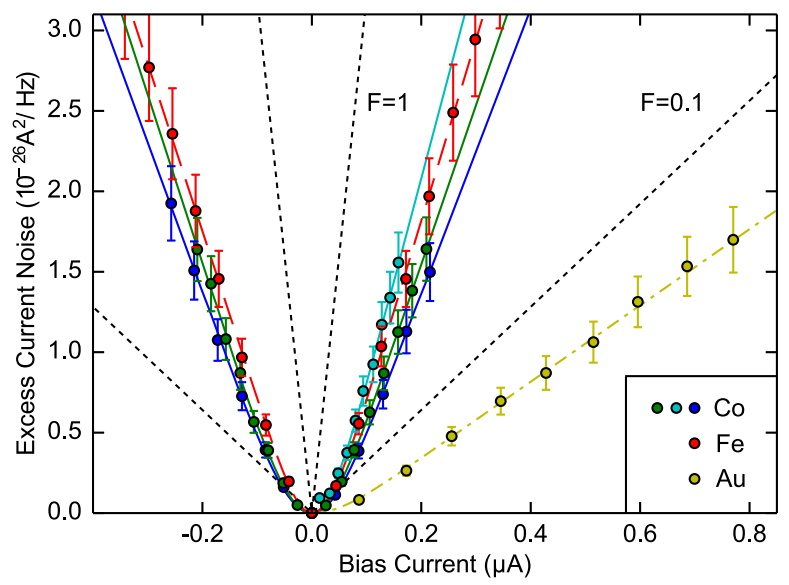

FIG. 3 (color online). Excess noise power vs bias current. Dashed, dash dotted, and solid lines are fits to the experimental data (circles) from $\mathrm{Fe}, \mathrm{Au}$, and three $\mathrm{Co}$ atoms. The Co data were acquired on different atoms and with different tips. Temperatures between 4.4 and $6 \mathrm{~K}$ were determined from the fits. Dotted lines show the excess noise at $F=1$ and $F=0.1$. 
Based on Ref. [24] the excess noise may be written as

$$
\Delta S=S-S_{\Theta}=F\left[S_{0} \operatorname{coth}\left(\frac{S_{0}}{S_{\Theta}}\right)-S_{\Theta}\right] .
$$

This expression was fit separately for positive and negative bias to the excess noise of Fig. 3 using the temperature $T$ (that determines $S_{\Theta}$ ) and the Fano factor $F$ as adjustable parameters. An excellent agreement with the experimental data is obtained.

Since $|V|$ was $<10 \mathrm{mV}$ during the noise measurements, the zero bias conductance determined from the thermal noise is used for the complete analysis. For $\mathrm{Co}(\mathrm{Fe})$ it was $24 \%(8 \%)$ smaller than the conductance at $V=128 \mathrm{mV}$. The former is due to the Kondo resonance.

Figure 4 shows an overview of the Fano factors and conductances determined from a series of measurements on stable $\mathrm{Fe}, \mathrm{Co}$, and $\mathrm{Au}$ contacts. For comparison, a solid curve depicts the minimum $F$ possible for spin-polarized transmission [cf. Eq. (1)]. A spin-degenerate transport with identical transparencies for both spin directions increases the minimum Fano factor to the dashed curve. The measurements on Au adatoms lie close to this curve, as observed in previous experiments $[6,7]$ and validate our experimental

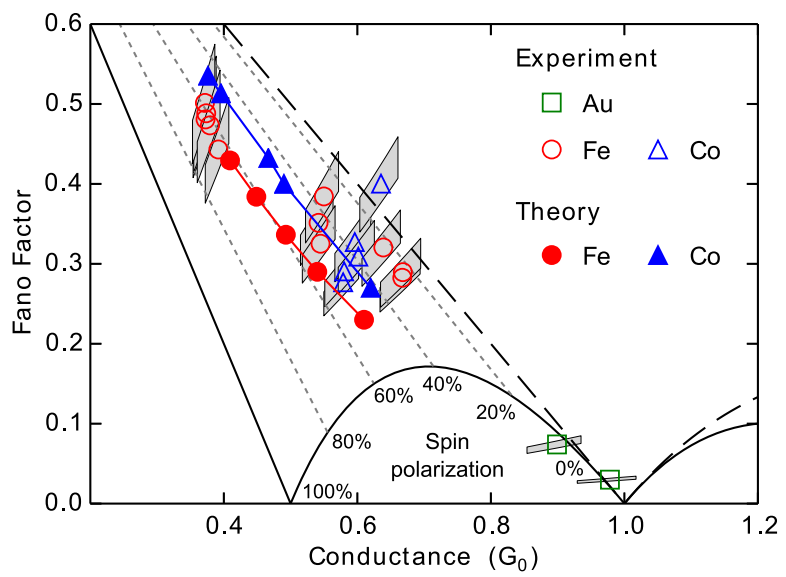

FIG. 4 (color online). Fano factors and zero bias conductances of $\mathrm{Au}, \mathrm{Fe}$, and $\mathrm{Co}$ adatom contacts determined from the excess and thermal noise densities, respectively. Solid and dashed lines show the smallest Fano factors possible for spin-polarized $\left(P=100 \%\right.$ up to $\left.0.5 G_{0}\right)$ and spin degenerate $(P=0 \%$ up to $1 G_{0}$ ) transport channels, respectively. Gray dotted lines indicate Fano factors in a single channel scenario for the indicated spin polarizations. Measurement uncertainties are indicated in gray. The experimental data for $\mathrm{Au}$ are consistent with a single spindegenerate channel, as expected. The Co data were recorded with different tips and on different atoms. Four out of five data sets indicate transport through a single spin-polarized channel. All data from $\mathrm{Fe}$ suggest single channel, spin-polarized transport. Five data sets around $0.38 G_{0}$ were recorded with the same tip on different atoms on a narrow terrace and reveal higher degrees of spin polarization. Theoretical data were calculated for tip-adatom distances (from left to right) of 4.10, 4.00, 3.95, 3.85, $3.70 \AA$ (Fe) and $4.25,4.20,4.10,4.05,3.85 \AA(\mathrm{Co})$. setup. The data from Co and Fe cluster between the solid and dashed lines. This behavior was observed from all contacts on Fe and Co, except for one Co data set which falls close to the dashed line. These combinations of Fano factors and conductances require spin-polarized transmission, possibly through a single dominating channel. We were surprised by this observation. First, in view of the high valency of $\mathrm{Co}$ and $\mathrm{Fe}$ atoms, single channel transport seems unlikely [25]. Second, it is intriguing to find a spin polarization in the case of a single magnetic adatom at a temperature as high as $4.5 \mathrm{~K}$. Yet while the current is spin polarized at any time, the results do not require the spin quantization axis to be fixed any longer than the tunneling time. Third, the similarly reduced noise for Co needs to be reconciled with the fact that Co displays a Kondo effect. Publications on the influence of the Kondo effect on shot noise are rare [26-28]. In general, a Kondo signature in the $d I / d V$ spectra does not imply that the impurity spin is completely screened. In an underscreened system, the ground state retains a spin degree of freedom and a spin polarization remains. While for single $\mathrm{Fe}$ atoms in $\mathrm{Au}$ and $\mathrm{Ag}$ crystals the number of screening channels is sufficient to quench the spin [29], Co atoms in molecules between two $\mathrm{Au}$ electrodes were shown to be underscreened [30]. Underscreening, possibly induced by surface anisotropy or the tip [31,32], may be at the origin of the noise reduction observed here.

To gain first insight into the microscopic transport mechanisms and to identify the relevant orbitals involved, we performed spin-polarized density functional theory

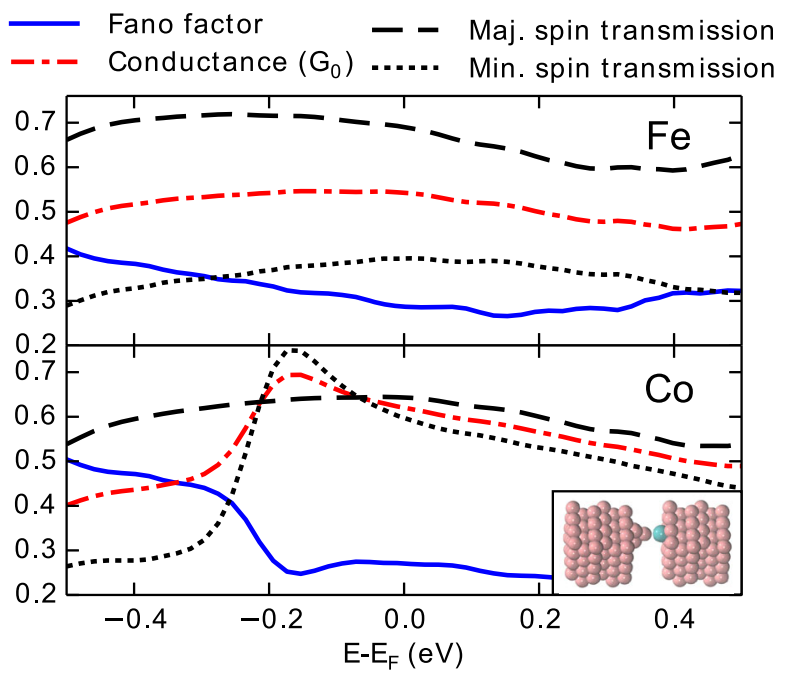

FIG. 5 (color online). Results of transport calculations for the structure shown in the inset for an Fe adatom (upper panel) and a Co adatom (lower panel) at $d=3.85 \AA$ using periodic boundary conditions in the surface. The energy-resolved conductances in units of $G_{0}, k$-point-averaged Fano factors and transmissions of majority and minority spin channels are shown as dash dotted, solid, dashed, and dotted lines. The values at $E_{F}$ should be compared to the experimental low-bias data. 
(DFT) calculations on the structure shown in Fig. 5 using the generalized gradient approximation exchangecorrelation potential [33] with a localized atomic orbital basis set (SIESTA) [34]. A $4 \times 4$ surface unit-cell and $3 \times 3$ surface $k$-point sampling were used. The linear conductance was subsequently calculated with the TRANSIESTA setup [35] using a $20 \times 20 k$-points average of both transmissions as well as the noise calculated from the eigenchannel transmissions [36], $\left\langle\tau_{i}(k)\left(1-\tau_{i}(k)\right)\right\rangle_{k}$. We varied the tip-sample distance to roughly match the obtained experimental conductances relaxing tip, adatom, and the outermost two surface layers. For Fe and Co distances of $d=$ $3.70 \ldots 4.10 \AA$ and $d=3.85 \ldots 4.25 \AA$ corresponding to $G=0.61 \ldots 0.41 G_{0}$ and $G=0.62 \ldots 0.38 G_{0}$ were used. The calculated conductances and Fano factors are included in Fig. 4 and match the experimental values.

Figure 5 shows the calculated spin channel transmissions $\left\langle\tau_{i, \sigma}\right\rangle_{k}$, along with $G$ and $F$ as a function of energy. We find for both $\mathrm{Fe}$ and $\mathrm{Co}$ that for each spin direction only one channel is dominating the transport process, while the transmissions of all others are suppressed by approximately 2 orders of magnitude. The dominating channel is spin polarized and rotationally symmetric around the tip axis ( $\Gamma_{1}$ symmetry) like the $s, p_{z}$, and $d_{z^{2}}$ orbitals of the adatom. Thus the Au atom at the tip apex acts as an orbital filter, as the coupling of its $s$ orbital to other $d$ and $p$ orbitals of the magnetic atom $\left(d_{x y}, d_{x z}, d_{y z}, d_{x^{2}-y^{2}}, p_{x}, p_{y}\right)$ is strongly suppressed by symmetry and would require a next-nearest neighbor coupling. This is also consistent with DFT calculations on $\mathrm{Cu} / \mathrm{Co} / \mathrm{Cu}$ contacts [38] that showed a dominating $\Gamma_{1}$ channel in the tunneling and weak contact regimes.

As the modeling of our systems confirmed a single relevant transport channel (per spin), the Fano factor can be used to determine the spin polarization of the current, $P=\left(\tau_{\uparrow}-\tau_{\downarrow}\right) /\left(\tau_{\uparrow}+\tau_{\downarrow}\right)$. Fano factors resulting from different values of $P$ are plotted in Fig. 4 as dotted lines. These lines also represent lower boundaries of the spin polarization in multichannel scenarios. The measured Fano factors for the $\mathrm{Co}$ and $\mathrm{Fe}$ atoms around $0.6-0.7 G_{0}$ result in spin polarizations of $30 \%-50 \%$. Larger spin polarizations of $\approx 60 \%$ (data sets around $0.38 G_{0}$ in Fig. 4) were measured with an identical tip on Fe atoms that were located in close proximity on a narrow terrace. The role of the tip, a possible magnetic coupling between the adatoms, and the influence of the local electronic structure of the substrate may be interesting to investigate in future experiments.

The method presented above may be useful for a variety of systems. The present results indicate that a stability of the localized spin over extended time intervals is not required.

We wish to thank J. Kröger and C. Barreteau for the discussions. Financial support via Sonderforschungsbereich 668 is acknowledged.
[1] The power spectral density $S$ used in this Letter is defined as the zero frequency limit of $2 \int_{-\infty}^{\infty} \mathrm{dt} e^{i \omega t}\langle\Delta I(0) \Delta I(t)\rangle$, where \langle\rangle indicates the ensemble average. For a formal definition, cf. [2].

[2] S. Kogan, Electronic Noise and Fluctuations in Solids, 1st ed. (Cambridge University Press, Cambridge, England, 1996).

[3] W. Schottky, Ann. Phys. (Berlin) 362, 541 (1918).

[4] T. Martin and R. Landauer, Phys. Rev. B 45, 1742 (1992).

[5] S. Heinze, M. Bode, A. Kubetzka, O. Pietzsch, X. Nie, S. Blügel, and R. Wiesendanger, Science 288, 1805 (2000).

[6] H. E. van den Brom and J. M. van Ruitenbeek, Phys. Rev. Lett. 82, 1526 (1999).

[7] M. Kumar, R. Avriller, A. L. Yeyati, and J. M. van Ruitenbeek, Phys. Rev. Lett. 108, 146602 (2012).

[8] Y. M. Blanter and M. Büttiker, Phys. Rep. 336, 1 (2000).

[9] H. Birk, M. J. M. de Jong, and C. Schönenberger, Phys. Rev. Lett. 75, 1610 (1995).

[10] M. Herz, S. Bouvron, E. Cavar, M. Fonin, W. Belzig, and E. Scheer, Nanoscale 5, 9978 (2013).

[11] U. Kemiktarak, T. Ndukum, K. C. Schwab, and K. L. Ekinci, Nature (London) 450, 85 (2007).

[12] R. Chen, P. J. Wheeler, M. Di Ventra, and D. Natelson, Sci. Rep. 4, 4221 (2014).

[13] R. Cron, M. F. Goffman, D. Esteve, and C. Urbina, Phys. Rev. Lett. 86, 4104 (2001).

[14] R. Vardimon, M. Klionsky, and O. Tal, Phys. Rev. B 88, 161404 (2013).

[15] M. Kiguchi, O. Tal, S. Wohlthat, F. Pauly, M. Krieger, D. Djukic, J. C. Cuevas, and J. M. van Ruitenbeek, Phys. Rev. Lett. 101, 046801 (2008).

[16] N. L. Schneider, G. Schull, and R. Berndt, Phys. Rev. Lett. 105, 026601 (2010).

[17] N. L. Schneider, J. T. Lü, M. Brandbyge, and R. Berndt, Phys. Rev. Lett. 109, 186601 (2012).

[18] M. Kumar, O. Tal, R. H. M. Smit, A. Smogunov, E. Tosatti, and J. M. van Ruitenbeek, Phys. Rev. B 88, 245431 (2013).

[19] V. Madhavan, W. Chen, T. Jamneala, M. F. Crommie, and N. S. Wingreen, Science 280, 567 (1998).

[20] A. Kumar, L. Saminadayar, D. C. Glattli, Y. Jin, and B. Etienne, Phys. Rev. Lett. 76, 2778 (1996).

[21] J. A. Stroscio and R. J. Celotta, Science 306, 242 (2004).

[22] A. Sperl, J. Kröger, and R. Berndt, Phys. Rev. B 81, 035406 (2010).

[23] N. Agraït, A. L. Yeyati, and J. M. van Ruitenbeek, Phys. Rep. 377, 81 (2003).

[24] G. B. Lesovik, Sov. Phys. JETP Lett. 49, 592 (1989).

[25] E. Scheer, N. Agraït, J. C. Cuevas, A. L. Yeyati, B. Ludoph, A. Martín-Rodero, G. R. Bollinger, J. M. van Ruitenbeek, and C. Urbina, Nature (London) 394, 154 (1998).

[26] T. Delattre, C. Feuillet-Palma, L. G. Herrmann, P. Morfin, J.-M. Berroir, G. Feve, B. Placais, D. C. Glattli, M.-S. Choi, C. Mora, and T. Kontos, Nat. Phys. 5, 208 (2009).

[27] E. Sela, Y. Oreg, F. von Oppen, and J. Koch, Phys. Rev. Lett. 97, 086601 (2006).

[28] O. Zarchin, M. Zaffalon, M. Heiblum, D. Mahalu, and V. Umansky, Phys. Rev. B 77, 241303(R) (2008).

[29] T. A. Costi, L. Bergqvist, A. Weichselbaum, J. von Delft, T. Micklitz, A. Rosch, P. Mavropoulos, P. H. Dederichs, F. Mallet, L. Saminadayar, and C. Bäuerle, Phys. Rev. Lett. 102, 056802 (2009). 
[30] J. J. Parks, A. R. Champagne, T. A. Costi, W. W. Shum, A. N. Pasupathy, E. Neuscamman, S. Flores-Torres, P. S. Cornaglia, A. A. Aligia, C. A. Balseiro, G. K.-L. Chan, H. D. Abruña, and D. C. Ralph, Science 328, 1370 (2010).

[31] N. Néel, J. Kröger, L. Limot, K. Palotas, W. A. Hofer, and R. Berndt, Phys. Rev. Lett. 98, 016801 (2007).

[32] N. Néel, J. Kröger, and R. Berndt, Phys. Rev. B 82, 233401 (2010).

[33] J. P. Perdew, K. Burke, and M. Ernzerhof, Phys. Rev. Lett. 77, 3865 (1996).

[34] J. M. Soler, E. Artacho, J. D. Gale, A. García, J. Junquera, P. Ordejón, and D. Sánchez-Portal, J. Phys. Condens. Matter 14, 2745 (2002).
[35] M. Brandbyge, J.-L. Mozos, P. Ordejón, J. Taylor, and K. Stokbro, Phys. Rev. B 65, 165401 (2002).

[36] The noise is $k$-point sampled and, therefore, not simply proportional to $T(1-T)$, where $T$ is the $k$-averaged spin channel transmission; cf. the Supplemental Material [37].

[37] See Supplemental Material at http://link.aps.org/ supplemental/10.1103/PhysRevLett.114.016602 for detailed description of the data acquisition, the data processing and the theoretical calculations.

[38] M. Polok, D. V. Fedorov, A. Bagrets, P. Zahn, and I. Mertig, Phys. Rev. B 83, 245426 (2011). 